\title{
Prolactin in rats is attenuated by conspecific touch in a novel environment
}

\author{
JANIE H. WILSON \\ Georgia Southern University, Statesboro, Georgia
}

\begin{abstract}
Prolactin is released in response to physical and psychological stress, the latter of which involves an animal's interpretation of its environment as potentially dangerous. This interpretation might be altered by social buffering, as defined by the presence of a social partner. In support of this claim, I previously found that the presence of a conspecific during exposure to the open field caused lower levels of prolactin in juvenile rats than did exposure to the open field alone. Because testing with a conspecific allowed the animals to touch, physical contact may have been necessary for the reduction of prolactin. To test this hypothesis, juvenile male and female rats in the present study were exposed to the open field (1) alone, (2) with a conspecific separated by a Plexiglas partition, or (3) with a conspecific without a partition. Touch was necessary to reduce stress as measured by prolactin levels.
\end{abstract}

Although generally known for its role in reproductive processes, prolactin is also released in response to acute stressors such as physical discomfort and psychological stress. Nursing animals have higher levels of prolactin than non-nursing animals, yet lactation is associated with less stress responding (Uvnäs-Moberg, 1998). Conversely, among virgin animals, high levels of prolactin are reliably associated with greater responses to stress challenge. In a recent review of prolactin, Freeman, Kanyicska, Lerant, and Nagy (2000) have identified two primary roles: reproductive processes and homeostasis, the latter of which encompasses an animal's response to stress. Prolactin released during stress activates the immune system and protects the animal from the potentially harmful effects of a stressful environment (Freeman et al., 2000).

Exposing an adult rat to an acute physical or psychological stressor results in activation of the hypothalamicpituitary-adrenal (HPA) axis, which involves release of ACTH followed by release of corticosterone, a hormone well known to be involved in stress responding. Although ACTH levels rapidly return to normal, corticosterone levels increase more slowly and remain high for a longer duration (Kant, Bauman, Anderson, \& Mougey, 1992). As corticosterone levels increase, prolactin levels decrease proportionately, indicating that corticosterone moderates prolactin (Abel, 1993). Similarly, adrenalectomized rats with corticosterone replacement have lower levels of prolactin than do those without corticosterone under both physical and psychological stressors (Yelvington, Weiss, \& Ratner, 1984).

This research was supported in part by Instrumentation and Laboratory Improvement Grant DUE-9751060 from the National Science Foundation. Correspondence should be addressed to J. H. Wilson, Department of Psychology, Georgia Southern University, Statesboro, GA 30460 (e-mail: jhwilson@gasou.edu).
In the rat literature, physical stressors are those that cause physical discomfort, including footshock (Yelvington et al., 1984), forced swim (Armario, LopezCalderon, Jolin, \& Balasch, 1986), and restraint (Briski \& Sylvester, 1987). Psychological stressors are defined by the animal's interpretation of the environment as potentially dangerous, and they include noise (Armario, Gil, Marti, Pol, \& Balasch, 1991), a conditioned-fearparadigm (Paris et al., 1987), and a novel environment such as an unfamiliar open field (Yelvington et al., 1984).

Although the vast majority of stress research has been conducted on stressful environments in which an animal is alone, stress can be induced in various social situations. Defensive aggression has been associated with increases in levels of prolactin in both male Wistar rats (Dijkstra, Tilders, Hiehle, \& Smelik, 1992) and Siberian dwarf hamsters (Castro \& Matt, 1997). Among humans, giving an oral presentation to an audience has been rated as stressful and was found to be related to higher levels of prolactin than the control group (Gerritsen, Heijnen, Weigant, Bermond, \& Frijda, 1996). Although these studies indicate that negative social situations are stressful as measured by prolactin levels, the current literature does not provide adequate coverage of the relationship between social stress and prolactin.

Whereas negative social situations increase stress, positive social conditions reduce stress, as measured by levels of cortisol/corticosterone and prolactin. Adult male and juvenile female squirrel monkeys in established social groups have been found to have lower levels of cortisol than have animals individually housed (Levine, Lyons, \& Schatzberg, 1997; Lyons, Ha, \& Levine, 1995). Similarly, adult male Siberian dwarf hamsters housed with a female or a female and pups have been found to have lower levels of prolactin than have males housed alone (Castro \& Matt, 1997). When adult male Wistar albino rats were group-housed or isolated for 5 weeks, iso- 
lated rats had higher levels of hypothalamic corticosterone and serum prolactin (Gambardella, Greco, Sticchi, Bellotti, \& Di Renzo, 1994). In the same species, Brown and Grunberg (1995) found that group housing of adult females reduced serum corticosterone below levels in individually housed females. When housing conditions became crowded, levels of corticosterone among females remained lower than individual housing, but males showed a significant increase in corticosterone, revealing an effect of sex on stress responses to housing (Brown \& Grunberg, 1995). Thus, given adequate housing space, less stress under group housing conditions is pervasive for both males and females across several species.

The benefits of affiliation are also evident when a stressful environment is experienced. Female squirrel monkeys exposed to a conditioned fear paradigm (CS paired with footshock) with conspecifics have been found to have lower cortisol levels than have those tested individually (Stanton, Patterson, \& Levine, 1985), although cortisol levels in the same species remained unchanged during exposure to a novel environment with and without a conspecific (Hennessy, 1986). Among humans experiencing a computer task for which they received false negative feedback, social support and encouragement were found to lower cortisol levels (Biondi et al., 1986). In a comparable experiment, humans receiving social support during a challenging computer task had lower levels of cortisol than did participants with no form of social support (Thorsteinsson, James, \& Gregg, 1998). Similarly, among human males, psychological stress induced by preparing to speak to an audience was reduced by social support from a girlfriend as measured by lower cortisol levels (Kirschbaum, Klauer, Filipp, \& Hellhammer, 1995). Biondi and Picardi (1999) have suggested that neuroendocrine responses to stress may be dictated, at least in part, by our interpretations of the event, and social support may offer a buffer against psychological stress. This explanation lends support to the idea that a stressful environment may be interpreted as less stressful when social support is available.

Just as cortisol levels are attenuated by the presence of a social partner during stress, two studies indicate that prolactin also decreases. Humans who received social support during negative feedback on a computer task had lower levels of prolactin than those who did not have social encouragement (Biondi et al., 1986). Similarly, I have recently found that rats acutely exposed to the stressful open field in the presence of a conspecific had lower prolactin levels than did those tested alone in the open field (Wilson, 2000). As with cortisol, one possible explanation for these results is that the psychological stress associated with the stressors was reduced by the presence of a social partner.

Although social buffering is a likely explanation for lower levels of prolactin in the presence of a conspecific, it was also noted that rats in the social condition were allowed physical contact as well as the visual, olfactory, and auditory information linked with touch (Wilson, 2000). The present study was designed for further inves- tigation of the components of the social environment that might reduce stress. Animals were tested alone (Group A), with the opportunity to touch a conspecific (Group T), or separated from the conspecific by a partition that allowed visual (clear Plexiglas), olfactory (small holes in the partition), and auditory (open ceiling) input (Group S). It was hypothesized that prolactin levels of rats allowed physical contact with a conspecific would be lower than those for animals separated by a partition and those tested alone. Rats separated by a partition were expected to have lower levels than would animals tested alone, revealing a social benefit from being in the presence of a conspecific even though touching could not occur.

\section{METHOD}

\section{Subjects}

Seventy (38 male and 32 female) juvenile Long-Evans hooded rats were utilized in this study. Rats were obtained by breeding animals from Harlan Sprague Dawley Laboratories; the day of birth was considered Postnatal Day (PD) 0. Litters were culled to 10 pups, and they remained with dams in Plexiglas cages $(45.72 \times 22.86 \times$ $20.32 \mathrm{~cm}$ ) with pine bedding until weaning. On PD 22, juveniles were weaned and individually housed in metal chambers $\left(19.05 \mathrm{~cm}^{2}\right)$ with a wire-mesh back and a removable Plexiglas front until the time of testing. The colony was on a 12:12-h reversed light:dark schedule, with the light phase beginning at 6:00 p.m. Food and water were available ad libitum.

\section{Behavioral Testing}

On PD 23, each rat was individually placed in a novel open field for $5 \mathrm{~min}$ to become habituated to the testing chamber. The open field was constructed of glass $(60.96 \times 30.48 \times 40.64 \mathrm{~cm})$ with $2 \mathrm{~cm}$ of pine bedding on the floor of the chamber. A removable segment of clear Plexiglas was used to separate the arena into two halves, with the ceiling of the arena remaining open. The Plexiglas partition, $.64 \mathrm{~cm}$ thick, contained 126 holes $(.32 \mathrm{~cm}$ in diameter) $1.27 \mathrm{~cm}$ apart in the lower $17.78 \mathrm{~cm}$ of the partition. During habituation, all animals were placed in the left side of the chamber. On PD 24, rats were randomly assigned to one of three testing conditions: (1) alone in the open field, (2) in the open field with a conspecific separated by a Plexiglas partition, or (3) in the open field with a conspecific without the partition. Owing to the diverse timing of parturitions, the animals were tested with same-sex conspecifics without control of whether or not they were littermates, although the majority of pairings were with littermates. Habituation and testing occurred between 11:00 a.m. and 2:30 p.m. under $120 \mathrm{~W}$ of red light.

A video camera recorded the behaviors of all animals for $10 \mathrm{~min}$ on the day of testing. Rats in Group A were always tested on the left side of the partitioned chamber; in Group S, the rat on the left side of the chamber was considered the experimental animal; and in Group T, pairs of rats were placed in the left side of the partitioned chamber. In Groups A and S, the number of times at the partition as well as the average length of time at the partition were recorded. In Groups $S$ and $T$, the number of times animals came together, either at the partition in Group S or touching in Group T, was recorded.

Within 1 min of open-field testing, each rat was decapitated. In Groups $S$ and T, trunk blood from the first rat decapitated was collected; blood from the second rat in the pair was not analyzed.

\section{Prolactin Assay}

Prolactin assays were completed using kits from ALPCO, with prolactin levels expressed in $\mathrm{ng} / \mathrm{ml}$ and assay sensitivity at $0.6 \mathrm{ng} / \mathrm{ml}$. Standard curves and samples were assayed in duplicate. Intra-assay variabilities for the two sets of samples were $15 \%$ and $1 \%$. Inter-assay variability was $2 \%$. 


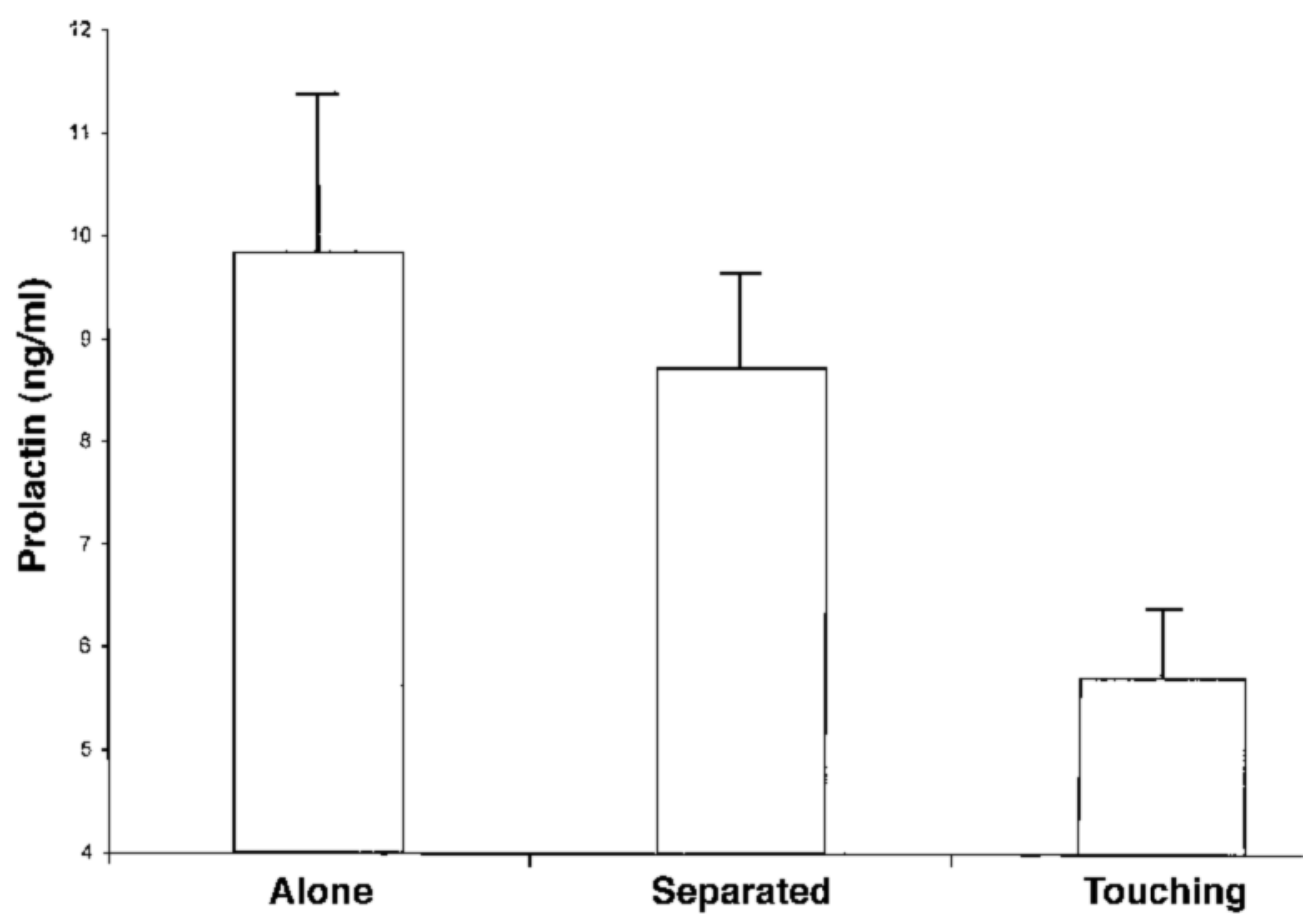

Figure 1. Animals were tested alone, with a conspecific separated by a partition, or with the opportunity to touch a conspecific. Rats allowed to touch had lower prolactin levels than did rats separated by a partition $(p<.05)$ and those tested alone $(p<.05)$. Rats separated from a conspecific and those tested alone did not differ in their levels of prolactin $(p>.05)$.

\section{RESULTS}

Prolactin was analyzed in a $2(\operatorname{sex}) \times 3$ (stress condition) analysis of variance (ANOVA). No main effect of sex or interaction between sex and stressor was found $(p>.05)$. However, stress condition affected prolactin levels $[F(2,64)=3.28, p<.05]$ (see Figure 1$)$. One-tailed, Fisher's protected $t$ tests were used to analyze the main effect of testing condition further. Animals allowed to touch a conspecific had significantly lower levels of prolactin $(M=5.71, S E M=.88)$ than did animals separated by a partition $(M=8.73, S E M=1.11)[t(64)=$ $1.83, p<.05]$, and those tested alone $(M=9.84, S E M=$ $1.49)[t(64)=2.45, p<.05]$. However, rats separated by a partition had prolactin levels similar to those of rats tested alone $[t(64)=.66, p>.05]$.

Behavioral data were submitted to 2 (sex) $\times 2$ (stress condition) ANOVAs. In Groups A and S, number of times at the partition as well as average length of time at the partition were analyzed. In Groups S and T, the number of times animals came together, either at the partition in Group S or touching in Group T, was recorded. Between Groups A and S, no main effect of sex or interaction between sex and stress condition was found $(p>.05)$. However, stress condition affected the number of times the animals came to the partition and the total amount of time rats were at the partition during the 10-min testing period $[F(1,42)=27.95, p<.001$, and $F(1,42)=30.12$, $p<.001$, respectively] (see Figure 2). Animals tested with a conspecific on the other side of the partition came to the partition more often $(M=21.17, S E M=1.30)$ than those tested alone $(M=12.41, S E M=.96)$. Conversely, animals separated from a conspecific spent less time (seconds) at the partition during the 10-min testing session $(M=144.22, S E M=11.05)$ than did rats tested alone $(M=261.77, S E M=18.37)$. Between Groups $S$ and $\mathrm{T}$, the number of times the animals came together (either touching in the $\mathrm{T}$ condition or at the same section of the partition in the $\mathrm{S}$ condition) did not yield a main effect for sex or an interaction between sex and stress condition ( $p>.05$ ). A main effect of group was found for the number of times animals came together and the total amount of time rats spent together $[F(1,44)=26.62, p<$ .001 , and $F(1,44)=93.43, p<.001$, respectively] (see Figure 3). Animals allowed to touch did so more often $(M=30.17, S E M=2.80)$ than separated animals came together at the partition $(M=14.25, S E M=1.06)$. In the same pattern, animals allowed to touch spent more time (seconds) together $(M=437.07, S E M=16.93)$ than did those separated by the partition $(M=217.97, S E M=$ 14.86).

Pearson's correlations between prolactin levels and behaviors did not reveal significant relationships $(p>.05)$, although animals separated by the partition tended to 

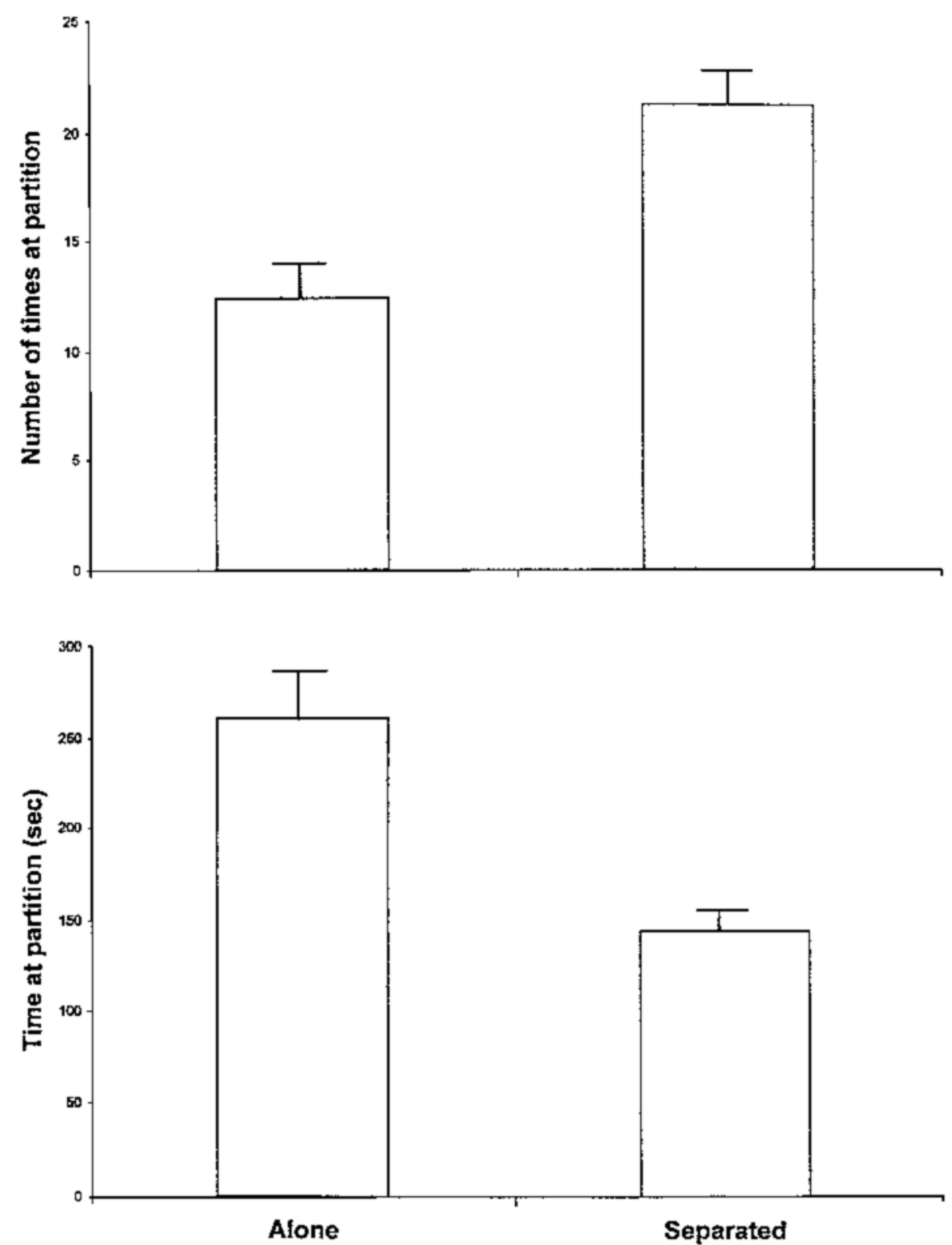

Figure 2. Animals separated by a partition came to the partition more often but left more quickly than those tested alone ( $p<.05$ for both analyses).

have lower levels of prolactin as the amount of time they came together at the partition increased $[r(24)=-.37$, $p=.08]$.

\section{DISCUSSION}

Animals allowed to touch experienced social buffering in a social situation as measured by lower prolactin levels than did those tested alone in the open field and those tested with a conspecific separated by a partition. Because the partition and testing arena allowed separated animals to experience the sensory inputs of vision, olfaction, and audition, these components do not appear to be sufficient to reduce stress in a novel open field. In a previous study indicating the importance of touch in reducing prolactin levels, hens were allowed to make physical contact with chicks, to see and hear chicks separated by a wire cage, or to only hear chick vocalizations from a nearby cage (Richard-Yris et al., 1998). Hens that made physical contact with chicks had lower levels of prolactin than did those allowed to see and hear or only hear chicks (Richard-Yris et al., 1998).

In the present study, animals separated from a conspecific returned to the partition more often but also left the partition more quickly than those tested alone with the partition in place in the chamber. One possible ex- 

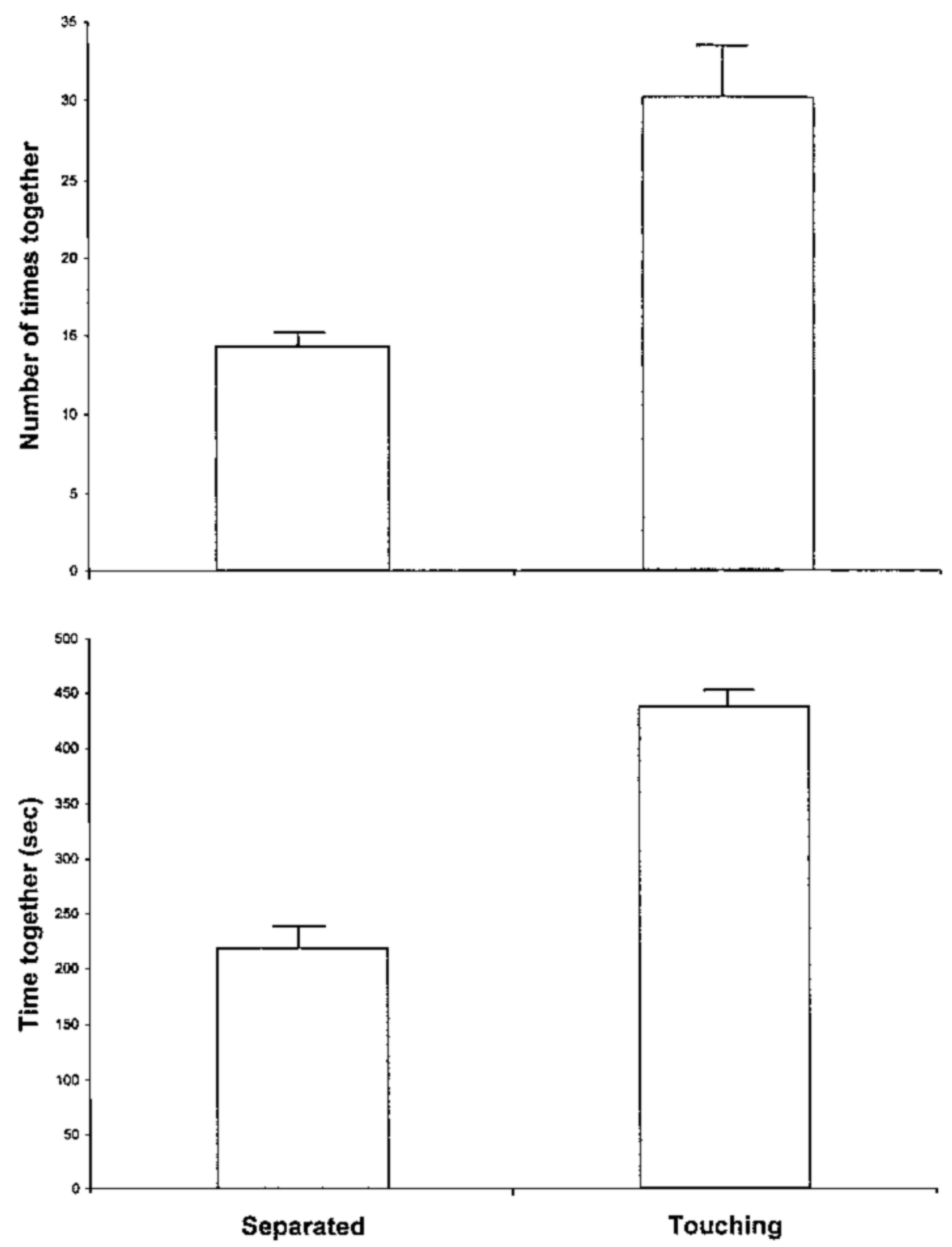

Figure 3. Animals allowed to touch came together more frequently and spent more time together than did those separated by the partition ( $p<.05$ for both analyses).

planation for these results is that rats separated from a conspecific were still drawn to the area closest to the conspecific; but lack of possible contact was realized, and the animal continued to quickly leave the area of the partition. Although the animal may have been motivated to seek contact, the most likely strategy to achieve contact, direct approach, was not effective.

Separate animals came to the partition in the same vicinity as the conspecific fewer times as compared with the number of times animals touched when tested together in the same compartment. In addition, separated animals spent less total time in the same region by the partition than did animals allowed physical contact. These results taken together are not surprising: Animals allowed to touch came together more often and spent more time together when they did touch. The reinforcement of touch is apparent, with animals seeking out touch as a social buffer against the psychological stress of the open field. In support of this explanation, Taylor (1981) found that adult male Long-Evans rats exposed to the psychological stressor of noise sought out other adult males in a T-maze more reliably than animals not exposed to noise stress. During training, the animals had learned that they would be allowed to interact (including touch) with the other male after they had chosen the conspecific as their goal, indicating that social contact—perhaps specifically 
touch-was reinforcing, especially under conditions of psychological stress.

It is interesting that prolactin and amount of time spent together were marginally related among animals separated by the partition, indicating that some benefit may have been obtained even when touching was not possible. In fact, analysis of prolactin and behavior only of animals allowed to touch did not reveal any significant relationships. This could have been due to a restriction-of-range problem: The majority of animals allowed to touch had low levels of prolactin.

Juvenile animals were used in this study, and play occurs quite often during this stage of development (Panksepp \& Beatty, 1980). Play has been defined by pins, which are characterized by the ventral surfaces of two rats touching, with the dorsal surface of one rat in contact with the floor (Panksepp \& Beatty, 1980). Indeed, animals in this study who were allowed to touch did pin during the 10 -min testing period $(M=18.67$, SEM $=$ 2.84) Thus, the benefits of touching seen in the present study may rely on the active component of play. Furthermore, the benefits of touch in reducing stress may be restricted to animals of this age regardless of the play component.

In the present study, animals were paired with samesex conspecifics, and the majority of those pairings occurred with littermates. Under these conditions, touch was beneficial; however, mixed-sex pairings as well as pairings with unfamiliar animals have not been examined in juveniles. Although the majority of the literature indicates that same-sex housing reduces stress relative to individual housing as measured by cortisol/corticosterone and prolactin levels, mixed-sex housing has yielded conflicting results. In adult male squirrel monkeys, pairing with a female and pairing with several females resulted in higher levels of cortisol than did housing with other males (Levine et al., 1997). However, human males had lower levels of cortisol when a female significant other was present under stressful conditions than did men tested alone, and females under stress did not benefit from the presence of their male significant others (Kirschbaum et al., 1995).

In most same-sex conditions, social groups were established well before testing began. Hennessy (1986) did not find a difference in the cortisol levels of adult female squirrel monkeys when they were exposed to a novel environment with a familiar partner or an unfamiliar partner. It should be noted that these groups did not differ from animals placed in the novel environment alone either, failing to support social buffering under stress regardless of familiarity. In humans, lower levels of cortisol have been found in males under stress who were supported by their female partners than in males supported by women who were strangers (Kirschbaum et al., 1995). However, females under the same stress who were supported by their partners tended to have higher levels of cortisol than did females supported by unfamiliar men, although the result did not reach significance at the .05 level $(p=.10)$.

The present study supports previous research which indicates that social buffering occurs in rats exposed to the open field in the presence of a conspecific (Wilson, 2000). An additional component now apparent is the importance of touch for social buffering to occur. Because animals in this study spent more time in physical contact than in the same vicinity at a clear partition, the behavioral data further support the suggestion that touching is more reinforcing than merely having visual, olfactory, and auditory input from a conspecific. The facets of touch necessary for social buffering under various psychological stressors warrant further investigation.

\section{REFERENCES}

ABEL, E. L. (1993). Physiological correlates of the forced swim test in rats. Physiology \& Behavior, 54, 309-317.

Armario, A., Gil, M., Marti, J., Pol, O., \& Balasch, J. (1991). Influence of various acute stressors on the activity of adult male rats in a holeboard and in the forced swim test. Pharmacology, Biochemistry \& Behavior, 39, 373-377.

Armario, A., Lopez-Calderon, A., Jolin, T., \& Balasch, J. (1986). Response of anterior pituitary hormones to chronic stress: The specificity of adaptation. Neuroscience \& Biobehavioral Reviews, 10, 245-250.

Biondi, M., Pancheri, P., Falaschi, D., Teodori, A., Paga, G., Delle Chiaie, R. DiCasare, G., \& Proietti, A. (1986). Social support as a moderator of the psychobiological stress response. New Trends in Experimental Clinical Psychiatry, 2, 173-183.

Biondi, M., \& PiCARDI, A. (1999). Psychological stress and neuroendocrine function in humans: The last two decades of research. Psychotherapy \& Psychosomatics, 68, 114-150.

Briski, K. P., \& Sylvester, P. W. (1987). Effects of repetitive daily acute stress on pituitary $\mathrm{LH}$ and prolactin release during exposure to the same stressor or a second novel stress. Psychoneuroendocrinology, 12, 429-437.

Brown, K. J., \& GrunberG, N. E. (1995). Effects of housing on male and female rats: Crowding stresses males but calms females. Physiology \& Behavior, 58, 1085-1089.

CASt RO, W. L. R., \& MATt, K. S. (1997). The importance of social condition in the hormonal and behavioral responses to an acute social stressor in the male Siberian dwarf hamster (Phodopus sungorus). Hormones \& Behavior, 32, 209-216.

Dijkstra, H., Tilders, F. J., Hiehle, M. A., \& Smelik, P. G. (1992). Hormonal reactions to fighting in rat colonies: Prolactin rises during defence, not during offence. Physiology \& Behavior, 51, 961-968.

Freeman, M. E., Kany icska, B., Lerant, A., \& Nagy, G. (2000). Prolactin: Structure, function, and regulation of secretion. Physiological Reviews, 80, 1523-1631.

Gambardella, P., Greco, A. M., Sticchi, R., Bellotti, R., \& Di Renzo, G. (1994). Individual housing modulates daily rhythms of hypothalamic catecholaminergic system and circulating hormones in adult male rats. Chronobiology International, 11, 213-221.

Gerrit sen, W., Heijnen, C. J., Weigant, V. M., Bermond, B., \& FriJda, N. H. (1996). Experimental social fear: Immunological, hormonal, and autonomic concomitants. Psychosomatic Medicine, 58, 273-286.

HenNessy, M. B. (1986). Effects of social partners on pituitary-adrenal activity during novelty exposure in adult female squirrel monkeys. Physiology \& Behavior, 38, 803-807.

Kant, G. J., Bauman, R A., Anderson, S. M., \& Mougey, E. H. (1992). Effects of controllable vs. uncontrollable chronic stress on stressresponsive plasma hormones. Physiology \& Behavior, 51, 1285-1288.

Kirschbaum, C., Klauer, T., Filipp, S. H., \& Hellhammer, D. H. (1995). Sex-specific effects of social support on cortisol and subjective 
responses to acute psychological stress. Psychosomatic Medicine, 57, 23-31.

Levine, S., Lyons, D. M., \& Schatzberg, A. F. (1997). Psychobiological consequences of social relationships. In C. S. Carter, I. I. Lederhendler, \& B. Kirkpatrick (Eds.), The integrative neurobiology of affiliation (Annals of the New York Academy of Sciences, Vol. 807, pp. 210-218). New York: New York Academy of Sciences.

Lyons, D. M., HA, C. M. G., \& Levine, S. (1995). Social effects and circadian rhythms in squirrel monkey pituitary-adrenal activity. Hormones \& Behavior, 29, 177-190.

PAnksePp, J., \& BeATty, W. W. (1980). Social deprivation and play in rats. Behavioral \& Neural Biology, 30, 197-206.

Paris, J. M., Lorens, S. A., Van de Kar, L. D., Urban, J. H., Richardson-Morton, K. D., \& Bethea, C. L. (1987). A comparison of acute stress paradigms: Hormonal responses and hypothalamic serotonin. Physiology \& Behavior, 39, 33-43.

Richard-Yris, M. A., Sharp, P. J., Wauters, A. M., Guemene, D., Richard, J. P., \& Foraste, M. (1998). Influence of stimuli from chicks on behavior and concentrations of plasma prolactin and luteinizing hormone in incubating hens. Hormones \& Behavior, 33, 139-148.
Stanton, M. E., Patterson, J. M., \& Levine, S. (1985). Social influences on conditioned cortisol secretion in the squirrel monkey. Psychoneuroendocrinology, 10, 125-134.

TAY LOR, G. T. (1981). Fear and affiliation in domesticated male rats. Journal of Comparative \& Physiological Psychology, 95, 685-693.

Thorsteinsson, E. B., James, J. E. \& GregG, M. E. (1998). Effects of video-relayed social support on hemodynamic reactivity and salivary cortisol during laboratory-based behavioral challenge. Health Psychology, 17, 436-444.

Uv Näs-MobERG, K. (1998). Oxy tocin may mediate the benefits of positive social interactions and emotions. Psychoneuroendocrinology, 23, 819-835.

Wilson, J. H. (2000). A conspecific attenuates prolactin responses to the open field. Hormones \& Behavior, 38, 39-43.

Yelvington, D. B., Weiss, G. K., \& Ratner, A. (1984). Effects of corticosterone on the prolactin response to psychological and physical stress in rats. Life Sciences, 35, 1705-1711.

(Manuscript received January 30, 2001; revision accepted for publication May 31, 2001.) 